\title{
Association between small dense low-density lipoprotein cholesterol and neuroimaging markers of cerebral small vessel disease in middle-aged and elderly Chinese populations
}

Xiaorong $\mathrm{Yu}^{\dagger}$, Yanhua $\mathrm{Yu}^{\dagger}$, Cunsheng Wei ${ }^{\dagger}$, Lin Wang, Junying Jiang, Rui Zhang, Qi Dai, Yue Kang and Xuemei Chen ${ }^{*}$

\begin{abstract}
Background: Cerebral small vascular disease (CSVD) is one of the leading causes of death in the aged population and is closely related to abnormalities in low-density lipoprotein cholesterol (LDL-C). Our study aims to clarify the relationship between small and dense low-density lipoprotein cholesterol (sdLDL-C) (a subcomponent of LDL-C) and neuroimaging markers of CSVD.

Methods: In total, 1211 Chinese adults aged $\geq 45$ years with cranial magnetic resonance imaging (MRI) were recruited in this retrospective study from January 2018 to May 2021. Serum lipids and other baseline characteristics were investigated in relation to the occurrence of CSVD. A logistic regression model was performed to analyze the relationships between LDL subtypes and CSVD risk, and the Pearson correlation coefficient was used to analyze the correlation between clinical characteristics and CSVD risk. ROC curves and AUCs were created and depicted to predict the best cutoff value of LDL-C subtypes for CSVD risk. Based on these data, we performed comprehensive analyses to investigate the risk factors for CSVD.

Results: Ultimately, 623 eligible patients were included in the present study. Of the 623 eligible patients, 487 were included in the CSVD group, and 136 were included in the group without CSVD (control group). We adjusted for confounders in the multivariate logistic regression model, and LDL-C3 was still higher in the CSVD patients than in the group of those without CSVD (OR $(95 \% \mathrm{Cl}), 1.22(1.08-1.38), P<0.05)$. Pearson correlation showed that there was a positive correlation between the levels of $L D L-C 3, L D L-C 4, L D L-C 5$, glucose, age, hypertension, previous ischemic stroke and CSVD risk $(r>0.15, P<0.01)$. Moreover, the best cutoff value of LDL-C3 to predict CSVD was $9.5 \mathrm{mg} / \mathrm{dL}$ with $68.4 \%$ sensitivity and $72.8 \%$ specificity, and the best cutoff value of LDL-C4 to predict CSVD was $5.5 \mathrm{mg} / \mathrm{dL}$ with $50.5 \%$ sensitivity and $90.4 \%$ specificity.
\end{abstract}

*Correspondence: 13347808579@189.cn

${ }^{+}$Xiaorong Yu, Yanhua Yu and Cunsheng Wei contributed equally to this

work.

Department of Neurology, The Affiliated Jiangning Hospital with Nanjing

Medical University, Nanjing 211100, Jiangsu, China

(C) The Author(s) 2021. Open Access This article is licensed under a Creative Commons Attribution 4.0 International License, which permits use, sharing, adaptation, distribution and reproduction in any medium or format, as long as you give appropriate credit to the original author(s) and the source, provide a link to the Creative Commons licence, and indicate if changes were made. The images or other third party material in this article are included in the article's Creative Commons licence, unless indicated otherwise in a credit line to the material. If material is not included in the article's Creative Commons licence and your intended use is not permitted by statutory regulation or exceeds the permitted use, you will need to obtain permission directly from the copyright holder. To view a copy of this licence, visit http://creativecommons.org/licenses/by/4.0/. The Creative Commons Public Domain Dedication waiver (http://creativeco mmons.org/publicdomain/zero/1.0/) applies to the data made available in this article, unless otherwise stated in a credit line to the data. 
Conclusion: The results indicate that LDL-C3 is an independent risk factor for CSVD. A new prediction model based on LDL-C3 and LDL-C4 can help clinicians identify high-risk CSVD, even in people with normal LDL-C levels. The levels of sdLDL-C should be considered in the assessment and management of CSVD.

Keywords: LDL subclasses, Small and dense LDL, Cerebral small vessel disease, Burden, MRI markers

\section{Background}

Cerebral small vascular disease (CSVD) is an imaging, pathological and clinical syndrome caused by intracranial small vessel disease [1,2], and it shows a high prevalence in the aged population [2-4]. CSVD is one of the leading causes of stroke outcome [5-7], cognitive impairment $[8,9]$, and several neurological symptoms $[10,11]$. Cranial magnetic resonance imaging (MRI) markers of CSVD include white matter hyperintensity (WMH), lacunes, lobar and deep cerebral microbleeds (CMBs), and enlarged perivascular spaces (PVSs) [1]. These MRI markers might reflect the vulnerability of individual brains to pathologic insults. Therefore, it is necessary to screen the risk factors associated with MRI markers of CSVD.

Atherosclerosis is well known to contribute to the occurrence and development of ischemic stroke and CSVD [12-14], which is closely associated with abnormalities in serum lipids and lipoproteins, including elevated triglyceride levels, reduced high-density lipoprotein (HDL) cholesterol concentrations and increased low-density lipoprotein cholesterol (LDL-C) concentrations [15]. Specifically, serum LDL-C elevation is a common manifestation of dyslipidemia and leads to atherosclerosis $[15,16]$. It is also an important risk factor for CSVD. Small and dense low-density lipoprotein cholesterol (sdLDL-C) is a subcomponent of LDL-C with small particles and high density and includes LDL-C3 to LDLC7 [16]. It is easily oxidized, cleared slowly and enters the arterial wall to cause atherosclerosis, which is harmful [17].

Previous studies have shown that sdLDL-C can be used as an independent marker of coronary heart disease [18, 19]. The risk assessment level of established risk factors is even better than that of traditional risk factor markers. In the CNS, sdLDL-C was associated with an increased risk of acute ischemic stroke (AIS), especially noncardioembolic stroke [19]. In addition, sdLDL-C was related to AIS severity and prognosis, suggesting the importance of sdLDL-C control in patients with neurological diseases [20]. However, little is known about the relationship between sdLDL-C and CSVD to further explore whether sdLDL-C plays a key role in the early stage of atherosclerosis in CSVD. If sdLDL-C is involved, the question arises as to whether it is a better indicator to predict the risk of CSVD in the general population.
Therefore, we aimed to undertake the present study to clarify the association between sdLDL-C and neuroimaging markers of CSVD.

\section{Methods \\ Study population}

The study group consisted of 1211 hospitalized patients due to dizziness or headaches from the Department of Neurology, the Affiliated Jiangning Hospital of Nanjing Medical University, between January 2018 and May 2021. The inclusion criteria were as follows: Aged $\geq 45$ years and cerebral MRI. The exclusion criteria were as follows: Participants with indications that may affect the evaluation of CSVD and those with severe head injury, defined as a head Abbreviated Injury Score (AIS) > 4 [21]; severe cerebral infarction was defined as the NIH Stroke Scale (NIHSS) $\geq 14$ [22]; severe cerebral hemorrhage, defined as baseline intraparenchymal hemorrhage volume $\geq 30 \mathrm{ml}$ or intraventricular hemorrhage [23]; or presence of multiple sclerosis, lacking complete clinical data, or brain malignancy. The study flowchart is shown in Fig. 1. Each subject signed the informed consent. The study protocols were approved by the Ethics Committee of Affiliated Jiangning Hospital of Nanjing Medical University.

\section{MRI acquisition}

MRI scanning was performed on a $3.0 \mathrm{~T}$ MRI scanner (Ingenia, Philips Medical Systems, the Netherlands) with an 8-channel receiver array headcoil. High-resolution T1-weighted axial images covering the whole brain were obtained by a 3D magnetization prepared rapid gradient-echo sequence: $\mathrm{TR}=8.1 \mathrm{~ms} ; \mathrm{FA}=90^{\circ}$; $\mathrm{TE}=3.7 \mathrm{~ms}$; FOV $=240 \times 240 \mathrm{~mm}$; acquisition matrix $=240 \times 222$; gap $=0 \mathrm{~mm}$, thickness $=1.0 \mathrm{~mm}$; number of slices $=170$. T2-weighted images were acquired using the following parameters: $\mathrm{TR}=4000 \mathrm{~ms} ; \mathrm{TE}=107 \mathrm{~ms} ; \mathrm{FA}=90^{\circ}$; FOV $=230 \times 230 \mathrm{~mm}$ acquisition matrix $=384 \times 384$; thickness $=1.5 \mathrm{~mm}$; gap $=0 \mathrm{~mm}$, number of slices $=18$. DWI-weighted images were obtained with the following: $\mathrm{TR}=2503 \mathrm{~ms} ; \mathrm{TE}=98 \mathrm{~ms} ; \mathrm{FA}=90^{\circ}$; $\mathrm{FOV}$ $=230 \times 230 \mathrm{~mm}$ acquisition matrix $=152 \times 122$; thickness $=1.5 \mathrm{~mm}$; gap $=0 \mathrm{~mm}$, number of slices $=18$. Susceptibility weighted imaging (SWI)-weighted images were acquired using the following parameters: $T R=16 \mathrm{~ms}$; $\mathrm{TE}=23 \mathrm{~ms} ; \mathrm{FA}=10^{\circ}$; FOV $=220 \times 180 \mathrm{~mm}$; acquisition matrix $=220 \times 180 ;$ thickness $=-0.6 \mathrm{~mm}$; gap $=0 \mathrm{~mm}$, 


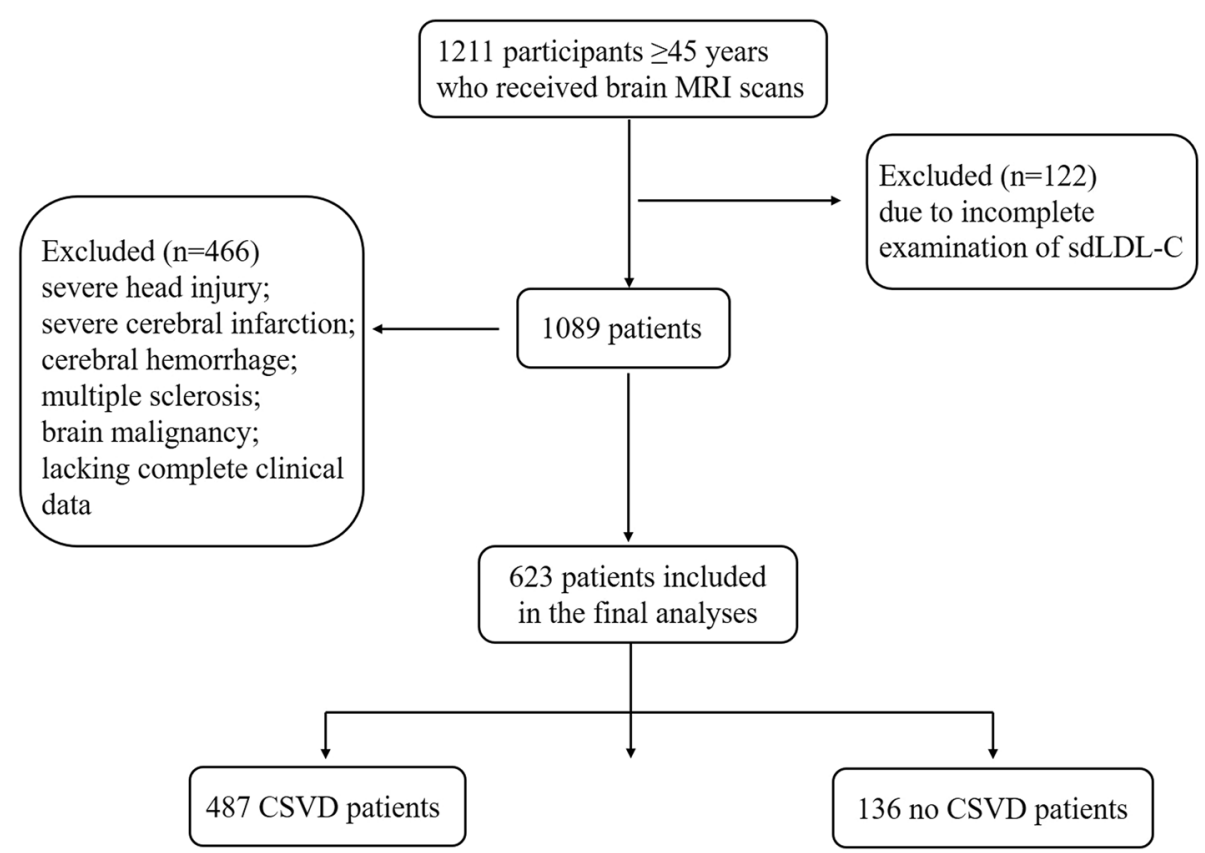

Fig. 1 Diagrammatic sketch of the screening process

number of slices $=200$. Additionally, T2 fluid-attenuated inversion recovery (FLAIR) axial images were obtained with the following parameters: $\mathrm{TR}=10,000 \mathrm{~ms}$; $\mathrm{TE}=120 \mathrm{~ms} ; \mathrm{FA}=110^{\circ} ; \mathrm{FOV}=220 \times 220 \mathrm{~mm}$; acquisition matrix $=336 \times 189$; thickness $=1.5 \mathrm{~mm}$; gap $=0 \mathrm{~mm}$, number of slices $=18$ [24].

\section{Fazekas score and CSVD burden score}

The results are reported in accordance with STRIVE [25]. The Fazekas score was used to score WMH [26]. We calculated the total CSVD score [27-29] using an ordinal scale (0 to 4 ) and counting the presence of each of the four MRI markers for CSVD (Fig. 2). Briefly, one point was awarded for each of the following items: Moderate to extensive $(10-25$ or $>25)$ PVS in the basal ganglia (1 point if present); $\geq 1$ lacune (1 point if present); periventricular WMH Fazekas score 3 or if deep WMH Fazekas score 2 or 3 (1 point if present); and $\geq 1$ deep CMB (1 point if present) [30]. The patients were divided into two groups according to their total CSVD score, that is, with CSVD (1-4 points) or without CSVD (0 points).

\section{Data collection}

The risk factors for cerebrovascular diseases were collected from each subject, including clinical and demographic information. The information consisted of age,
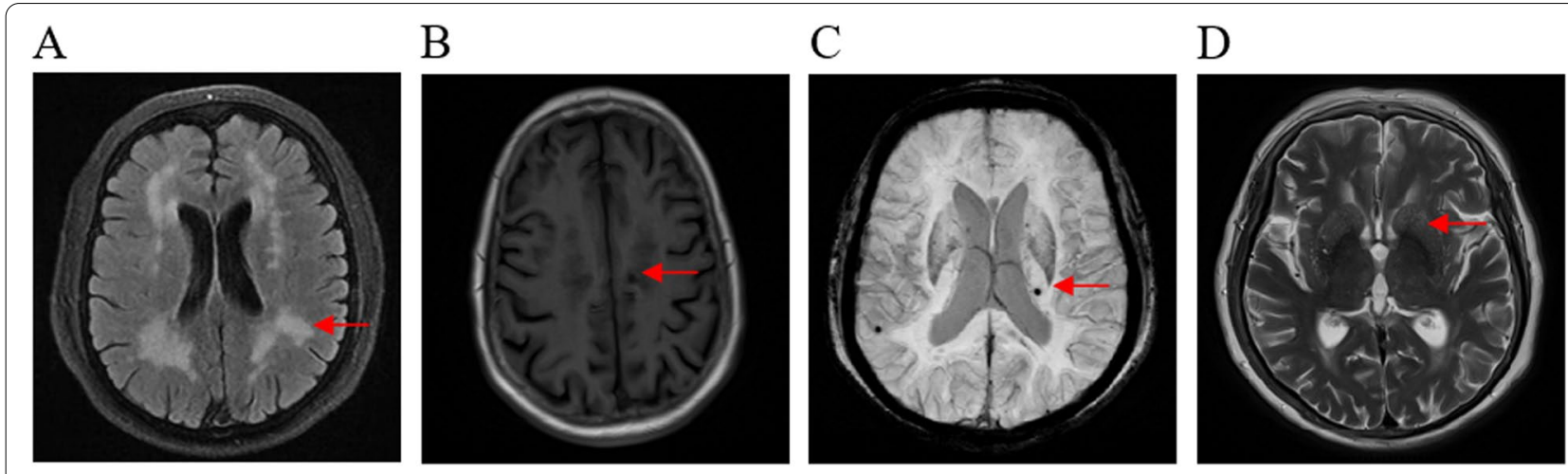

Fig. 2 MRI manifestations of cerebral small vessel disease 
sex, body mass index (BMI), medical history, and past or present cigarette or alcohol use. In addition, laboratory tests were carried out to determine fasting blood glucose (FBG), glycosylated hemoglobin (HbA1c), D-dimer levels, pulse pressure difference, total cholesterol (TC), triglyceride (TG), low-density lipoprotein (LDL), lipoprotein a $[\mathrm{Lp}(\mathrm{a})]$, lipoprotein-associated phospholipase A2 (Lp-PLA2), high-sensitivity C-reactive protein (H-CRP), uric acid (UA), and creatinine $(\mathrm{Cr})$. All the samples were collected from the venous blood of the subjects in a 12-h fasted state.

\section{Low-density lipoprotein analysis}

Blood samples were also used for LDL subfraction analysis. LDL subgroups were classified and measured with the Lipoprint System (Quantimetrix Corporation, Redondo Beach, CA, USA) according to the manufacturer's instructions as previously described [31, 32]. This system separates LDL subfractions by a polyacrylamide gel electrophoresis technique, and gels were scanned to determine the relative area of each lipoprotein subfraction after electrophoresis. Based on net surface charge and size, various stained LDL subfractions are classified according to their mobilities in the gel. By this analysis, LDL was divided into 7 subfractions (LDL-1 to LDL-7) [33]. LDL-1 and LDL-2 are defined as larger LDLs, and LDL3-7 are defined as sdLDL-C according to their size and density.

\section{Statistical analysis}

SPSS software (Version 22.0) was applied to analyze the data. Categorical variables are shown as numbers combined with percentages (\%). Continuous variables are shown as the mean \pm standard deviation (SD). The correlation of clinical variables with the risk of CSVD was analyzed by Pearson correlation analysis. For the univariate analysis, differences in clinical data were analyzed by Student's $t$-test and chi-square tests. To adjust for traditional risk factors, multivariable logistic regression analysis was performed by binary logistic regression analysis, which allowed adjustment for confounding factors. Optimum cutoff values were defined based on their highest diagnostic accuracy according to the $R O C$ curves. $p<0.05$ was considered statistically significant $(* p<0.05, * * p<0.01)$.

\section{Results}

\section{Characteristics of the subjects}

Ultimately, 623 eligible patients were included in the present study. Of the 623 eligible patients, 487 were included in the CSVD group, and 136 were included in the group without CSVD (control group). The mean ages of the CSVD patients and controls were $65.13 \pm 9.98$ and $60.38 \pm 9.31$ years old, with male ratios of 53.39 and
$42.65 \%$, respectively. The baseline characteristics of both study groups are shown in Table 1 . The age, pulse pressure difference, proportion of male patients, hypertension, diabetes, previous ischemic stroke, fasting plasma glucose (FPG), HbA1c, creatinine, UA, LDL-C, and LpPLA2 were significantly higher in the CSVD group than in the control group $(P<0.05)$. LDL-C1, LDL-C3, LDLC4, LDL-C5 and LDL-C6 showed statistically significant differences between the groups, with $P<0.01$ (Fig. 3). BMI, coronary artery disease, atrial fibrillation, previous cerebral hemorrhage, current smokers, current alcohol users, CRP, $D$-dimer levels, Lp(a), TG, TC and LDL-C7 did not differ between the two groups $(P \geq 0.05)$, while the HDL-C concentration was significantly higher in the control group $(P<0.05)$.

\section{Relationship of LDL subtypes with CSVD risk}

A logistic regression model was performed to analyze the relationship between LDL subtypes and CSVD risk. CSVD was used as a response variable, and risk factors were used as explanatory variables. Detailed results are shown in Table 2. LDL-C3 was significantly higher in the CSVD patients than in those without CSVD (OR (95\% CI), 1.18(1.06-1.32), $P<0.05)$. There was no significant difference among the CSVD group and the controls in terms of LDL-C, LDL-C1, LDL-C2, LDL-C4, LDL-C5, LDL-C6, and LDL-C7 $(P \geq 0.05)$.

In addition, we made further adjustments for LDL subtypes, sex, age, pulse pressure, hypertension, previous ischemic stroke, diabetes and statin therapy in the multivariate logistic regression model (Table 3). LDL-C3 was still higher in the CSVD patients than in those without CSVD (OR (95\% CI), 1.22(1.08-1.38), $P<0.05$ ). Moreover, gender, age, hypertension and previous ischemic stroke showed statistically significant differences between the two groups (OR (95\% CI), 0.62(0.40-0.99), 1.05(1.02$1.08), 2.04(1.26-3.31)$, and 1.60(0.85-3.00), respectively).

\section{Correlation between clinical characteristics and CSVD risk}

The Pearson correlation coefficient was used to analyze the correlation between clinical characteristics and CSVD risk (Fig. 4). There was a positive correlation between the levels of LDL-C3, LDL-C4, LDL-C5, glucose, age, hypertension, previous ischemic stroke and CSVD risk ( $\mathrm{r}>0.15, P<0.01)$. However, the LDL-C1 level, HDL and CSVD risk were negatively correlated $(r=-0.175$, $r=-0.116, P<0.01)$.

\section{Predictive value of LDL-C subtypes for CSVD risk}

To further evaluate the predictive values of LDL-C1, LDL-C2, LDL-C3, LDL-C4, LDL-C5, LDL-C6 and LDL-C7 in patients with CSVD, ROC curves and AUCs were created and depicted (Fig. 5, Table 4). LDL-C3 
Table 1 Clinical Characteristics at Baseline $(n=623)$ for patients with and without CSVD

\begin{tabular}{|c|c|c|c|}
\hline Variables & with CSVD $(n=487)$ & without CSVD $n=136$ ) & $P$ value \\
\hline Age, $y$, mean $\pm S D$ & $65.13 \pm 9.98$ & $60.38 \pm 9.31$ & 0.000 \\
\hline Male, n (\%) & 260(53.39) & $58(42.65)$ & 0.027 \\
\hline $\mathrm{BMI}, \mathrm{kg} / \mathrm{m}^{2}$, mean $\pm \mathrm{SD}$ & $26.82 \pm 31.69$ & $24.15 \pm 3.08$ & 0.309 \\
\hline Pulse pressure, $\mathrm{mmHg}$, mean $\pm \mathrm{SD}$ & $51.67 \pm 20.75$ & $45.70 \pm 21.47$ & 0.004 \\
\hline Hypertension, n (\%) & $311(64.79)$ & $53(39.55)$ & 0.000 \\
\hline Diabetes, n (\%) & 109(22.71) & 17(12.69) & 0.011 \\
\hline Coronary artery disease, $\mathrm{n}(\%)$ & $38(7.92)$ & $8(5.97)$ & 0.449 \\
\hline Atrial fibrillation, n (\%) & $3(0.63)$ & $0(0.00)$ & 0.359 \\
\hline Previous cerebral hemorrhage, $\mathrm{n}(\%)$ & 15(3.13) & $1(0.75)$ & 0.126 \\
\hline Previous ischemic stroke, n (\%) & 179(37.29) & 24(17.91) & 0.000 \\
\hline Current smokers, n (\%) & $77(16.04)$ & $20(14.93)$ & 0.754 \\
\hline Current alcohol user, n (\%) & $65(13.54)$ & 12(8.96) & 0.156 \\
\hline $\mathrm{CRP}(\mathrm{mg} / \mathrm{L})$, mean $\pm \mathrm{SD}$ & $4.06 \pm 22.07$ & $4.01 \pm 17.03$ & 0.981 \\
\hline$D$-dimer levels, $\mathrm{mg} / \mathrm{L}$, mean $\pm \mathrm{SD}$ & $0.55 \pm 1.98$ & $0.38 \pm 0.42$ & 0.369 \\
\hline $\mathrm{FPG}, \mathrm{mmol} / \mathrm{L}$, mean $\pm \mathrm{SD}$ & $5.81 \pm 2.09$ & $5.34 \pm 1.44$ & 0.018 \\
\hline $\mathrm{HbA} 1 \mathrm{C}, \%$, mean $\pm \mathrm{SD}$ & $5.99 \pm 1.26$ & $5.59 \pm 0.88$ & 0.002 \\
\hline creatinine, $\mu \mathrm{mol} / \mathrm{L}$, mean $\pm S D$ & $64.95 \pm 20.57$ & $60.07 \pm 14.28$ & 0.011 \\
\hline Uric acid, $\mu \mathrm{mol} / L$, mean $\pm S D$ & $311.94 \pm 85.93$ & $294.61 \pm 80.56$ & 0.040 \\
\hline $\mathrm{Lp}(\mathrm{a}), \mathrm{mg} / \mathrm{L}$, mean $\pm \mathrm{SD}$ & $243.57 \pm 230.55$ & $272.18 \pm 274.72$ & 0.098 \\
\hline Total cholesterol, mean \pm SD & $4.40 \pm 1.14$ & $4.24 \pm 1.17$ & 0.136 \\
\hline Triglyceride, mean $\pm S D$ & $1.98 \pm 1.67$ & $2.31 \pm 1.96$ & 0.062 \\
\hline $\mathrm{LDL}-\mathrm{C}, \mathrm{mmol} / \mathrm{L}$, mean $\pm \mathrm{SD}$ & $2.59 \pm 0.84$ & $2.39 \pm 0.84$ & 0.018 \\
\hline $\mathrm{LDL}-\mathrm{C} 1, \mathrm{mg} / \mathrm{dl}$, mean $\pm \mathrm{SD}$ & $24.30 \pm 13.48$ & $30.14 \pm 13.92$ & 0.000 \\
\hline $\mathrm{LDL}-\mathrm{C} 2, \mathrm{mg} / \mathrm{dl}$, mean $\pm \mathrm{SD}$ & $23.73 \pm 10.35$ & $23.04 \pm 11.19$ & 0.498 \\
\hline $\mathrm{LDL}-\mathrm{C} 3, \mathrm{mg} / \mathrm{dl}$, mean $\pm \mathrm{SD}$ & $14.02 \pm 8.11$ & $8.40 \pm 6.50$ & 0.000 \\
\hline $\mathrm{LDL}-\mathrm{C} 4, \mathrm{mg} / \mathrm{dl}$, mean $\pm \mathrm{SD}$ & $7.55 \pm 6.99$ & $2.69 \pm 4.05$ & 0.000 \\
\hline $\mathrm{LDL}-\mathrm{C} 5, \mathrm{mg} / \mathrm{dl}$, mean $\pm \mathrm{SD}$ & $2.94 \pm 4.82$ & $0.56 \pm 1.73$ & 0.000 \\
\hline $\mathrm{LDL}-\mathrm{C} 6, \mathrm{mg} / \mathrm{dl}$, mean $\pm \mathrm{SD}$ & $0.45 \pm 1.57$ & $0.08 \pm 0.39$ & 0.006 \\
\hline $\mathrm{LDL}-\mathrm{C} 7, \mathrm{mg} / \mathrm{dl}$, mean $\pm \mathrm{SD}$ & $0.10 \pm 0.57$ & $0.06 \pm 0.34$ & 0.440 \\
\hline $\mathrm{HDL}-\mathrm{C}, \mathrm{mmol} / \mathrm{L}$, mean $\pm \mathrm{SD}$ & $1.12 \pm 0.28$ & $3.25 \pm 16.12$ & 0.005 \\
\hline Statin therapy, n (\%) & $242(50.42)$ & $60(44.78)$ & 0.248 \\
\hline $\begin{array}{l}\text { Lipoprotein-associated phospholipase A2, ng/mL, } \\
\text { mean } \pm S D\end{array}$ & $137.26 \pm 23.83$ & $140.26 \pm 16.81$ & 0.027 \\
\hline
\end{tabular}

Continuous variables are shown as the mean \pm standard deviation (SD), categorical variables are shown as numbers combined with percentage (\%). Pulse pressure means the difference between the systolic and diastolic pressures

$B M I$ Body mass index, CRP C-reactive protein, FPG fasting plasma glucose, HbA1C glycated hemoglobin, $L D L-C$ indicates low-density lipoprotein cholesterol; Lp(a), lipoprotein(a)

and LDL-C4 showed larger areas under the curves than the other subtypes of LDL-C (AUC, 0.718 and 0.730, respectively). Moreover, the best cutoff value of LDLC3 to predict CSVD was $9.5 \mathrm{mg} / \mathrm{dL}$ with $68.4 \%$ sensitivity and $72.8 \%$ specificity, and the best cutoff value of LDL-C4 to predict CSVD was $5.5 \mathrm{mg} / \mathrm{dL}$ with $50.5 \%$ sensitivity and $90.4 \%$ specificity.

\section{Discussion}

The main findings of this study were as follows: 1 ) even after adjustments for multiple confounding factors, including age, hypertension and previous ischemic stroke at baseline, higher baseline serum LDL-C3 levels were associated with an increased risk of CSVD; and 2) LDL-C3 and LDL-C4 can predict CSVD risk.

It is generally known that CSVD is a highly prevalent disease in older individuals. CSVD burden may signify a diminished capacity of cerebral tissue to withstand ischemia and could be a poor prognostic marker for stroke survivors. Therefore, there is continuous interest in identifying novel risk factors for CSVD burden. It is well known that age and hypertension are the most important risk factors for CSVD. Consistent with previous studies, we also found that age, hypertension and 


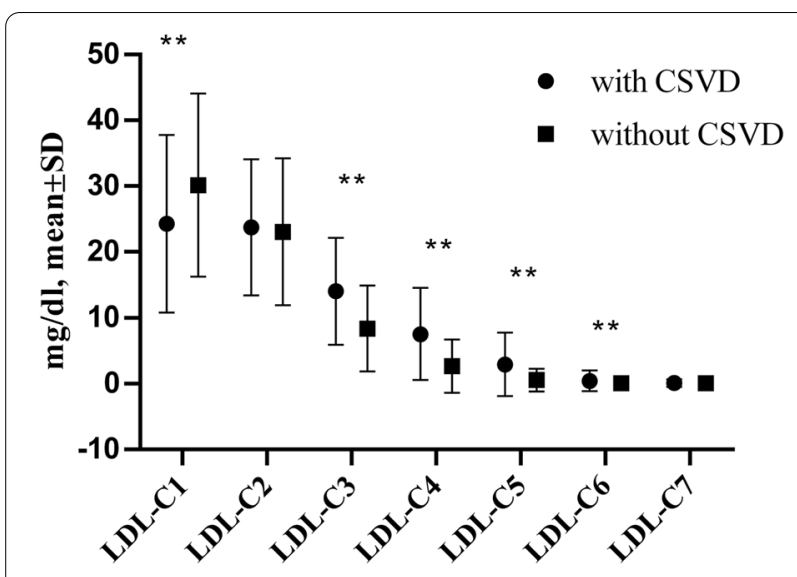

Fig. 3 The level of LDL subtypes for patients with and without CSVD

Table 2 The logistic regression analysis of LDL subtype for patients with and without CSVD

\begin{tabular}{lllll}
\hline Variables & $\boldsymbol{\beta}$ & Wals & OR $(\mathbf{9 5 \% C l )}$ & $\boldsymbol{P}$ value \\
\hline LDL-C1 & 0.010 & 0.646 & $1.01(0.99-1.03)$ & 0.422 \\
LDL-C2 & -0.047 & 3.801 & $0.95(0.91-1.00)$ & 0.051 \\
LDL-C3 & 0.169 & 8.680 & $1.18(1.06-1.32)$ & 0.003 \\
LDL-C4 & -0.094 & 1.044 & $0.91(0.76-1.09)$ & 0.307 \\
LDL-C5 & 0.268 & 3.595 & $1.31(0.99-1.73)$ & 0.058 \\
LDL-C6 & -0.102 & 0.120 & $0.90(0.51-1.60)$ & 0.729 \\
LDL-C7 & -0.367 & 0.234 & $0.86(0.45-1.61)$ & 0.628 \\
\hline
\end{tabular}

Model 1 additionally adjusted for LDL subtypes

$O R$ odds ratio, $\mathrm{Cl}$ confidence interval

Table 3 The logistic regression analysis of LDL subtype for patients with and without CSVD

\begin{tabular}{lllll}
\hline Variables & $\boldsymbol{\beta}$ & Wals & OR (95\%Cl) & $\boldsymbol{P}$ value \\
\hline LDL-C1 & 0.033 & 5.934 & $1.03(1.01-1.06)$ & 0.015 \\
LDL-C2 & -0.056 & 4.393 & $0.95(0.90-1.00)$ & 0.036 \\
LDL-C3 & 0.198 & 10.040 & $1.22(1.08-1.38)$ & 0.002 \\
LDL-C4 & -0.088 & 0.792 & $0.92(0.75-1.11)$ & 0.373 \\
LDL-C5 & 0.291 & 3.736 & $1.34(1.00-1.80)$ & 0.053 \\
LDL-C6 & -0.114 & 0.121 & $0.89(0.47-1.70)$ & 0.728 \\
LDL-C7 & 0.015 & 0.002 & $1.02(0.54-1.92)$ & 0.964 \\
Gender & -0.471 & 4.069 & $0.62(0.40-0.99)$ & 0.044 \\
Age & 0.047 & 12.885 & $1.05(1.02-1.08)$ & 0.000 \\
Pulse pressure & 0.008 & 1.904 & $1.01(1.00-1.02)$ & 0.168 \\
Hypertension & 0.714 & 8.377 & $2.04(1.26-3.31)$ & 0.004 \\
Previous ischemic stroke & 0.785 & 7.436 & $2.19(1.25-3.86)$ & 0.006 \\
Diabetes & 0.469 & 2.138 & $1.60(0.85-3.00)$ & 0.144 \\
Statin therapy & -0.263 & 1.242 & $0.77(0.48-1.22)$ & 0.265 \\
\hline
\end{tabular}

Model 2 adjust for LDL subtypes, Gender, Age, Pulse pressure, Hypertension, Previous ischemic stroke, Diabetes and Statin therapy

$O R$ odds ratio, $\mathrm{Cl}$ confidence interval previous ischemic stroke were associated with the burden of CSVD in our study population.

Another important traditional risk factor is the elevation of LDL-C, and LDL-C is commonly present concurrently with relevant pathologies, including large artery atherosclerosis or CSVD. However, an increasing number of studies have found that a high proportion of patients with atherosclerosis or acute vascular events have plasma cholesterol and LDL-C levels within the normal range [34]. This shows that LDL-C levels may not fully reflect the biological effect of LDL and the real blood lipid level of patients.

Recently, studies have confirmed that LDL-C consists of a series of particles with different sizes, densities, physicochemical compositions, metabolic behaviors and atherogenicity [35]. Specifically, LDL-1 to LDL-7 were defined by gradient gel electrophoresis. Among them, LDL-3 to LDL-7 are also named small dense LDL (sdLDL-C) according to their small size and high density, whereas LDL-1 and LDL-2 are defined as large LDLs. According to the National Cholesterol Education Program Adult Treatment Panel III (NCEP III), a high level of sdLDL-C is an established risk factor for cardiovascular disease (CVD) [36], and sd-LDL is recognized as the most important vascular contributor [37]. However, their clinical significance for CSVD is unclear.

Although abnormalities in serum lipids and lipoproteins have long been identified as risk factors for CSVD, TC, TG, Lp(a) and LDL-C in both groups were within the normal reference range in both CSVD patients and controls, suggesting that they are not the best biomarkers for CSVD prediction. Multivariable logistic regression confirmed that LDL-C3 was an independent variable of CSVD after adjustment for traditional vascular risk factors. Additionally, the LDL-C3 and LDL-C4 AUCs for CSVD were superior to those of other lipids, which indicated that the levels of these two serum sdLDL-Cs had better risk prediction for CSVD than other lipids, including LDL-C. The mechanisms related to the atherogenicity of sdLDL-C have long been recognized and include penetrating the arterial wall more easily, being more susceptible to oxidation or binding to glycosaminoglycans in the arterial wall, having a decreased binding ability to LDL-C receptors, and possessing an increased plasma residence time; thus, several studies have stated that sdLDL-C is an independent and more potent risk factor for coronary and peripheral artery disease as well as carotid atherosclerosis than LDL-C [38, 39].

Additionally, the correlation of LDL subfractions and the relationship of sd-LDL and serum lipids were assessed in our study. Consistent with the lower distribution of LDL-1 in the CSVD group, LDL-1 was negatively correlated with the sdLDL-C particles LDL-3 to 


\begin{tabular}{|c|c|c|}
\hline LDL-C4 & 0.297 & 1 \\
\hline LDL-C3 & 0.286 & \\
\hline LDL-C5 & 0.221 & \\
\hline Hypertension & 0.212 & \\
\hline Age & 0.196 & \\
\hline Previous ischemic stroke & 0.17 & \\
\hline $\mathrm{HbA} 1 \mathrm{C}$ & 0.137 & \\
\hline Pulse pressure & 0.117 & \\
\hline LDL-C6 & 0.11 & \\
\hline Creatinine & 0.104 & \\
\hline Diabetes & 0.102 & \\
\hline FPG & 0.097 & \\
\hline LDL-C & 0.095 & \\
\hline Lipoprotein-associated phospholipase A2 & 0.095 & \\
\hline Uric acid & 0.084 & \\
\hline Previous cerebral hemorrhage & 0.062 & 0 \\
\hline Total cholesterol & 0.059 & \\
\hline Current alcohol user & 0.057 & \\
\hline BMI & 0.04 & \\
\hline D-dimer levels & 0.038 & \\
\hline Atrial fibrillation & 0.037 & \\
\hline Coronary artery disease & 0.031 & \\
\hline LDL-C 7 & 0.031 & \\
\hline LDL-C2 & 0.027 & \\
\hline Current smokers & 0.013 & \\
\hline CRP & 0.001 & \\
\hline $\mathrm{Lp}(\mathrm{a})$ & -0.049 & \\
\hline Male & -0.092 & \\
\hline HDL-C & -0.116 & \\
\hline LDL-C1 & -0.175 & -1 \\
\hline
\end{tabular}

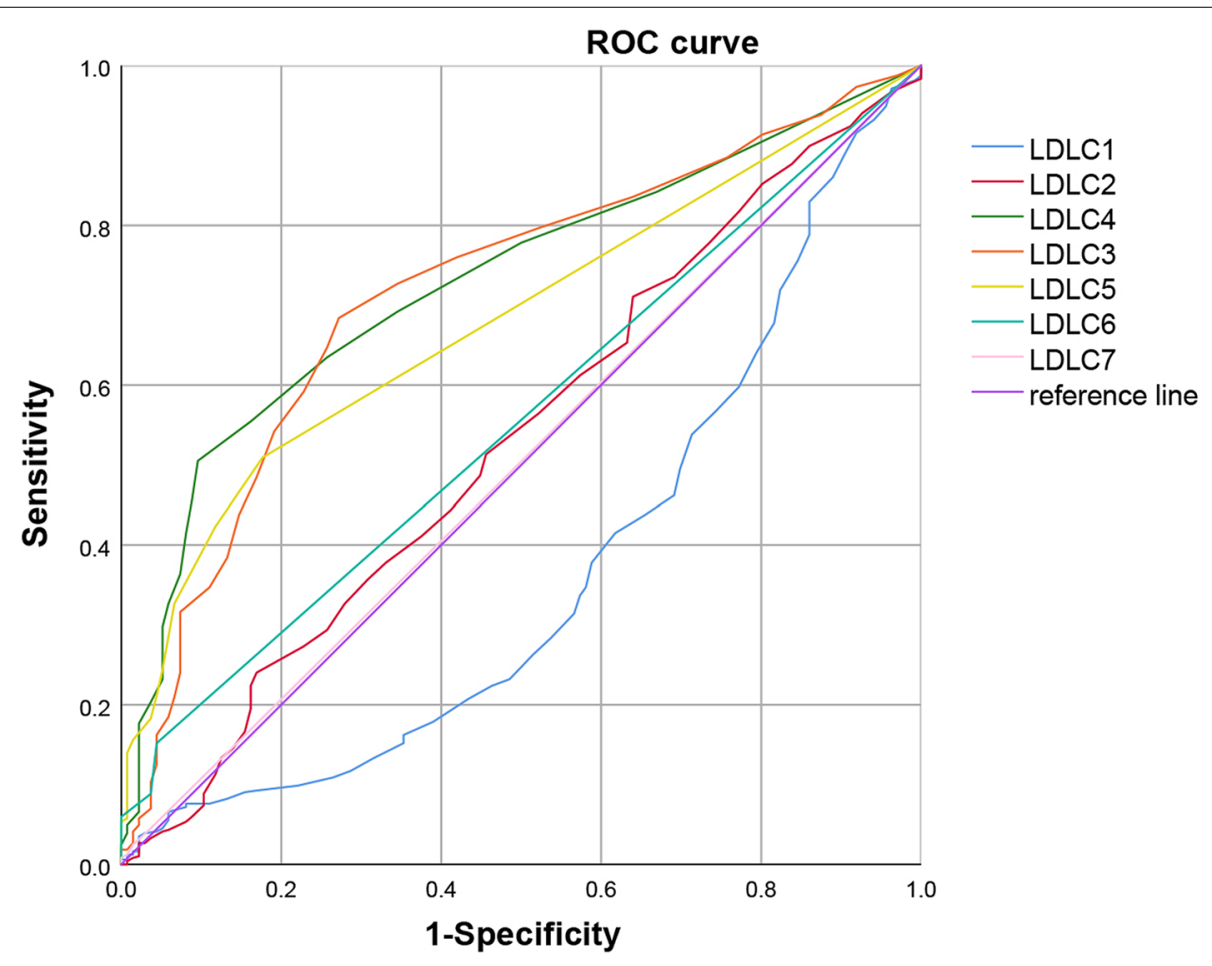

Fig. 5 Predictive Value of LDL-C subtypes for CSVD Risk 
Table 4 The area under ROC curve

\begin{tabular}{lllllllll}
\hline Variables & LDL-C & LDL-C1 & LDL-C2 & LDL-C3 & LDL-C4 & LDL-C5 & LDL-C6 & LDL-C7 \\
\hline Area & 0.579 & 0.365 & 0.531 & 0.718 & 0.730 & 0.681 & 0.554 & 0.504 \\
\hline
\end{tabular}

LDL-7, suggesting a lower potential atherogenicity of this particle. Considering the correlation coefficients of LDL-1 with LDL-C, it is highly recommended that not only LDL-C levels but also LDL subfractions (LDL-3 to LDL-7) should be taken into consideration for the management of CSVD. Generally, sdLDL-C is often accompanied by increased TG and TC and decreased HDL levels. In our study, there was a significant positive correlation between sdLDL-C levels and serum lipids, including TC, LDL-C, and TG, which is consistent with the findings reported by other studies [40].

Taken together, these observations may suggest that in patients with normal LDL-C levels, we need to consider other factors, such as sdLDL-C, to determine the risk of CSVD. Most importantly, a new prediction model was generated, which can help clinicians identify high-risk CSVD patients so that proper prevention measures can be taken to ease the potential burden and reduce suffering. Further, some evidence suggests that the presence and progression of cerebral atrophy is another potentially relevant manifestation of CSVD [41, 42], further study regarding the association between cerebral atrophy and sdLDL-C is warranted. Finally, whether neuropsychological alterations are a predictor of subcortical vascular dementia in the medium term and the relationship between sdLDL-C and silent lacunes on neuroimaging will be further explored in our future follow-up studies.

\section{Study strengths and limitations}

This is the first report demonstrating that sdLDL-C is an independent risk factor for increased CSVD after additional adjustment for other traditional risk factors. However, there are some limitations in the current study. First, the sample size of the participants enrolled was relatively small. Second, because of the nature of the retrospective research design, incomplete data collection, and lack of follow-up of prognosis, only relevant conclusions can be drawn without mechanistic research. Third, this study involved individuals in the Han population in the same region. Fourth, the effects of inflammatory markers on atherosclerosis were not considered in this study. In addition, we only collected the history of statins, but not the history of fibrates and other lipid-lowering drugs. Finally, previous studies have revealed that half of the patients with a first-ever lacunar infarct have mild cognitive impairment with subcortical vascular features and its presence may be a predictor of subcortical vascular dementia in the medium-long term [43], we did not evaluate cognitive function in the present study. Therefore, a large sample and prospective research are needed to overcome the above limitations.

\section{Abbreviations}

AIS: Acute ischemic stroke; BMI: Body mass index; CMB: Lobar and deep cerebral microbleed; CNS: Central nervous system; Cr: Creatinine; CSF: Cerebrospinal fluid; CSVD: Cerebral small vascular disease; CVD: Cardiovascular disease; DWI: Diffusion weighted imaging; FBG: Fasting blood glucose; FLAIR: Fluid-attenuated inversion recovery; HbA1c: Glycosylated hemoglobin; H-CRP: High-sensitivity C-reactive protein; IMT: Intima-media thickness; LDL: Low-density lipoprotein; Lp(a): Lipoprotein a; Lp-PLA2: Lipoprotein-associated phospholipase A2; MRI: Magnetic resonance imaging; PVS: Perivascular space; sdLDL-C: Small and dense low-density lipoprotein cholesterol; SWI: Susceptibility-weighted imaging; TC: Total cholesterol; TG: Triglyceride; UA: Uric acid; WMH: White matter hyperintensity; AIS: Abbreviated Injury Score; NIHSS: NIH Stroke Scale.

\section{Supplementary Information}

The online version contains supplementary material available at https://doi. org/10.1186/s12883-021-02472-6.

Additional file 1: Supplements Table 1. The correlation between clinical characteristics and severity of CSVD. Supplements Figure 1. The level of LDL subtypes between different total CSVD scores. Supplements Figure 2. The distribution of each component (WMH, CMBS, EPVS, and lacunes) of CSVD.

\section{Acknowledgments}

Not applicable.

Authors' contributions

XMC contributed to the study design. Data collection was performed by LW, JYJ, YK and RZ. YHY, QD, and XRY were responsible for data analysis. The manuscript was written by XMC and CSW. All authors approved the final manuscript for publication.

\section{Funding}

This research was supported by the Nanjing Medical Science and Technique Development Foundation (QRX17032, YKK20203); the Clinical Medical Science and Technology Development Fund of Jiangsu University (JLY2021153); the Kangda College of Nanjing Medical University Science and Research Development Project (KD2019KYJJYB047, KD2020KYJJZD010); and the Nanjing Medical University Science and Technology Development Project (NMUB2019240).

Availability of data and materials

Study data are available from the corresponding author upon request.

\section{Declarations}

\section{Ethics approval and consent to participate}

The present study is part of a longitudinal study on the pathogenesis of CSVD and the key techniques of clinical evaluation from Nanjing Drum Tower Hospital, the Affiliated Hospital of Nanjing University Medical School. 
We obtained ethical approval for this study from the ethics committee of the Affiliated Jiangning Hospital of Nanjing Medical University and performed in accordance with the Declaration of Helsinki (reference number, 2016YFC1300500). Written informed consent was obtained from all study participants.

\section{Consent for publication \\ Not applicable.}

\section{Competing interests}

None.

Received: 27 August 2021 Accepted: 22 October 2021

Published online: 09 November 2021

\section{References}

1. Wardlaw J, Smith C, Dichgans M. Mechanisms of sporadic cerebral small vessel disease: insights from neuroimaging. Lancet Neurol. 2013;12(5):483-97. https://doi.org/10.1016/s1474-4422(13)70060-7.

2. Siejka T, Srikanth V, Hubbard R, Moran C, Beare R, Wood A, et al. White matter Hyperintensities and the progression of frailty-the Tasmanian study of cognition and gait. J Gerontol A Biol Sci Med Sci. 2020;75(8):1545-50. https://doi.org/10.1093/gerona/glaa024.

3. Zhou Y, Gao H, Zhao F, Liang Y, Gao Y, Liu X, et al. The study on analysis of risk factors for severity of white matter lesions and its correlation with cerebral microbleeds in the elderly with lacunar infarction. Medicine. 2020;99(4):e18865. https://doi.org/10.1097/md.0000000000018865.

4. Molloy C, Nugent S, Bokde A. Alterations in diffusion measures of white matter integrity associated with healthy aging. J Gerontol A Biol Sci Med Sci. 2021;76(6):945-54. https://doi.org/10.1093/gerona/glz289.

5. Zerna C, Yu A, Modi J, Patel S, Coulter J, Smith E, et al. Association of White Matter Hyperintensities with Short-Term Outcomes in patients with minor cerebrovascular events. Stroke. 2018;49(4):919-23. https://doi.org/ 10.1161/strokeaha.117.017429.

6. Georgakis M, Duering M, Wardlaw J, Dichgans M. WMH and long-term outcomes in ischemic stroke: a systematic review and meta-analysis. Neurology. 2019;92(12):e1298-e308. https://doi.org/10.1212/wnl.00000 00000007142

7. Debette S, Schilling S, Duperron M, Larsson S, Markus H. Clinical significance of magnetic resonance imaging markers of vascular brain injury: a systematic review and Meta-analysis. JAMA Neurol. 2019;76(1):81-94. https://doi.org/10.1001/jamaneurol.2018.3122.

8. Knopman D, Griswold M, Lirette S, Gottesman R, Kantarci K, Sharrett A, et al. Vascular imaging abnormalities and cognition: mediation by cortical volume in nondemented individuals: atherosclerosis risk in communitiesneurocognitive study. Stroke. 2015;46(2):433-40. https://doi.org/10.1161/ strokeaha.114.007847.

9. van den Berg E, Geerlings M, Biessels G, Nederkoorn P, Kloppenborg R. White matter Hyperintensities and cognition in mild cognitive impairment and Alzheimer's disease: a domain-specific Meta-analysis. J Alzheimers Dis. 2018:63(2):515-27. https://doi.org/10.3233/jad-170573.

10. Pinter D, Ritchie S, Doubal F, Gattringer T, Morris Z, Bastin M, et al. Impact of small vessel disease in the brain on gait and balance. Sci Rep. 2017;7:41637. https://doi.org/10.1038/srep41637.

11. van Agtmaal M, Houben A, Pouwer F, Stehouwer C, Schram M. Association of Microvascular Dysfunction with Late-Life Depression: a systematic review and Meta-analysis. JAMA Psychiatry. 2017;74(7):729-39. https:// doi.org/10.1001/jamapsychiatry.2017.0984.

12. Liu Z, Zhao Y, Wang X, Zhang H, Cui Y, Diao Y, et al. Low carotid artery wall shear stress is independently associated with brain white-matter hyperintensities and cognitive impairment in older patients. Atherosclerosis. 2016;247:78-86. https://doi.org/10.1016/j.atherosclerosis.2016.02.003.

13. Nam K, Kwon H, Jeong H, Park J, Kim S, Jeong S, et al. Cerebral white matter hyperintensity is associated with intracranial atherosclerosis in a healthy population. Atherosclerosis. 2017;265:179-83. https://doi.org/10. 1016/j.atherosclerosis.2017.09.010

14. Chen Y, Hu M, Gong H. Correlation analysis between the LDL-C in serum and the onset of transient ischemic attack caused by CSVD. Exp Ther Med. 2017;14(2):1119-25. https://doi.org/10.3892/etm.2017.4583.
15. Rizzo M, Pernice V, Frasheri A, Di Lorenzo G, Rini G, Spinas G, et al. Small, dense low-density lipoproteins (LDL) are predictors of cardio- and cerebro-vascular events in subjects with the metabolic syndrome. Clin Endocrinol. 2009;70(6):870-5. https://doi.org/10.1111/j.1365-2265.2008. 03407.x.

16. Huh H, Park B, Lee S, Kang E, Park H. Establishing reference intervals for LDL subfractions in a Korean population using the Lipoprint LDL system. Clin Chem Lab Med. 2013;51(8):e179-82. https://doi.org/10.1515/ cclm-2012-0737.

17. Hoogeveen R, Gaubatz J, Sun W, Dodge R, Crosby J, Jiang J, et al. Small dense low-density lipoprotein-cholesterol concentrations predict risk for coronary heart disease: the atherosclerosis risk in communities (ARIC) study. Arterioscler Thromb Vasc Biol. 2014;34(5):1069-77. https://doi.org/ 10.1161/atvbaha.114.303284.

18. Zhang H, Jin J, Cao Y, Liu H, Zhang Y, Guo Y, et al. Association of small dense LDL-cholesterol with disease severity, hypertension status and clinical outcome in patients with coronary artery disease. J Hypertens. 2021;39(3):511-8. https://doi.org/10.1097/hjh.0000000000002678.

19. Sakai K, Koba S, Nakamura Y, Yokota Y, Tsunoda F, Shoji M, et al. Small dense low-density lipoprotein cholesterol is a promising biomarker for secondary prevention in older men with stable coronary artery disease. Geriatr Gerontol Int. 2018;18(6):965-72. https://doi.org/10.1111/ggi. 13287.

20. Zhou P, Liu J, Wang L, Feng W, Cao Z, Wang P, et al. Association of Small Dense Low-Density Lipoprotein Cholesterol with stroke risk, severity and prognosis. J Atheroscler Thromb. 2020;27(12):1310-24. https://doi.org/10. 5551/jat.53132.

21. Savitsky B, Givon A, Rozenfeld M, Radomislensky I, Peleg K. Traumatic brain injury: it is all about definition. Brain Inj. 2016;30(10):1194-200. https://doi.org/10.1080/02699052.2016.1187290.

22. Dhamoon MS, Moon YP, Paik MC, Boden-Albala B, Rundek T, Sacco RL, et al. Quality of life declines after first ischemic stroke. The Northern Manhattan Study. Neurology. 2010;75(4):328-34. https://doi.org/10.1212/ WNL.0b013e3181ea9f03.

23. Morgenstern LB, Zahuranec DB, Sánchez BN, Becker KJ, Geraghty M, Hughes R, et al. Full medical support for intracerebral hemorrhage. Neurology. 2015;84(17):1739-44. https://doi.org/10.1212/wnl.0000000000 001525.

24. Chen X, Wang L, Jiang J, Gao Y, Zhang R, Zhao X, et al. Association of neuroimaging markers of cerebral small vessel disease with short-term outcomes in patients with minor cerebrovascular events. BMC Neurol. 2021;21(1):21. https://doi.org/10.1186/s12883-021-02043-9.

25. Wardlaw J, Smith E, Biessels G, Cordonnier C, Fazekas F, Frayne R, et al. Neuroimaging standards for research into small vessel disease and its contribution to ageing and neurodegeneration. Lancet Neurol. 2013;12(8):822-38. https://doi.org/10.1016/s1474-4422(13)70124-8.

26. Fazekas F, Chawluk J, Alavi A, Hurtig H, Zimmerman R. MR signal abnormalities at 1.5 T in Alzheimer's dementia and normal aging. AJR Am J Roentgenol. 1987;149(2):351-6. https://doi.org/10.2214/ajr.149.2.351.

27. Staals J, Makin S, Doubal F, Dennis M, Wardlaw J. Stroke subtype, vascular risk factors, and total MRI brain small-vessel disease burden. Neurology. 2014;83(14):1228-34. https://doi.org/10.1212/wnl.0000000000000837.

28. Kwon H, Lynn M, Turan T, Derdeyn C, Fiorella D, Lane B, et al. Frequency, risk factors, and outcome of coexistent small vessel disease and intracranial arterial stenosis: results from the stenting and aggressive medical Management for Preventing Recurrent Stroke in intracranial stenosis (SAMMPRIS) trial. JAMA Neurol. 2016;73(1):36-42. https://doi.org/10.1001/ jamaneurol.2015.3145.

29. Huijts M, Duits A, van Oostenbrugge R, Kroon A, de Leeuw P, Staals J. Accumulation of MRI markers of cerebral small vessel disease is associated with decreased cognitive function. A Study in First-Ever Lacunar Stroke and Hypertensive Patients. Front Aging Neurosci. 2013;5:72. https://doi.org/10.3389/fnagi.2013.00072.

30. Chen X, Wang J, Shan Y, Cai W, Liu S, Hu M, et al. Cerebral small vessel disease: neuroimaging markers and clinical implication. J Neurol. 2019;266(10):2347-62. https://doi.org/10.1007/s00415-018-9077-3.

31. Hoefner D, Hodel S, O'Brien J, Branum E, Sun D, Meissner I, et al. Development of a rapid, quantitative method for $L D L$ subfractionation with use of the Quantimetrix Lipoprint LDL system. Clin Chem. 2001;47(2):266-74.

32. Varady K, Lamarche B. Lipoprint adequately estimates LDL size distribution, but not absolute size, versus polyacrylamide gradient gel 
electrophoresis. Lipids. 2011;46(12):1163-7. https://doi.org/10.1007/ s11745-011-3611-8.

33. Duan $\mathrm{R}$, Xue W, Wang K, Yin N, Hao H, Chu H, et al. Estimation of the LDL subclasses in ischemic stroke as a risk factor in a Chinese population. BMC Neurol. 2020;20(1):414. https://doi.org/10.1186/s12883-020-01989-6.

34. Castelli W. The new pathophysiology of coronary artery disease. Am J Cardiol. 1998;82:60T-5T. https://doi.org/10.1016/s0002-9149(98)00729-2.

35. Albers J, Kennedy H, Marcovina S. Evaluation of a new homogenous method for detection of small dense LDL cholesterol: comparison with the LDL cholesterol profile obtained by density gradient ultracentrifugation. Clin Chim Acta. 2011;412:556-61. https://doi.org/10.1016/j.cca.2010. 12.003.

36. National Cholesterol Education Program (NCEP) Expert Panel on Detection, Evaluation, and Treatment of High Blood Cholesterol in Adults (Adult Treatment Panel III). Third Report of the National Cholesterol Education Program (NCEP) Expert Panel on Detection, Evaluation, and Treatment of High Blood Cholesterol in Adults (Adult Treatment Panel III) final report. Circulation. 2002;106(25):3143-421.

37. Wang X, Wang L, Cao R, Yang X, Xiao W, Zhang Y, et al. Correlation between small and dense low-density lipoprotein cholesterol and cardiovascular events in Beijing community population. J Clin Hypertension (Greenwich). 2021;23(2):345-51. https://doi.org/10.1111/jch.14150.

38. Zeljkovic A, Vekic J, Spasojevic-Kalimanovska V, Jelic-Ivanovic Z, BogavacStanojevic N, Gulan B, et al. LDL and HDL subclasses in acute ischemic stroke: prediction of risk and short-term mortality. Atherosclerosis. 2010;210(2):548-54. https://doi.org/10.1016/j.atherosclerosis.2009.11.040.
39. Kayran Y, Yayla V, Çabalar M, Bajrami A, Karamanli Y, Gedikbaşi A, et al. LDL subclasses in ischemic stroke: a risk factor? Noro Psikiyatri Arsivi. 2019;56(1):13-7. https://doi.org/10.5152/npa.2017.19393.

40. Yazdandoust S, Parizadeh S, Moohebati M, Yaghmaei P, Rahsepar A, Tavallaie $S$, et al. Serum small dense low-density lipoprotein concentrations are elevated in patients with significant coronary artery stenosis and are related to features of the metabolic syndrome. Lipids. 2012;47(10):96372. https://doi.org/10.1007/s11745-012-3706-x.

41. Smith EE, Arboix A. Focal cortical thinning is caused by remote subcortical infarcts: spooky action at a distance. Neurology. 2012;79(20):2016-7. https://doi.org/10.1212/WNL.0b013e3182749f6e.

42. Wong FCC, Yatawara C, Low A, Foo H, Wong BYX, Lim L, et al. Cerebral small vessel disease influences hippocampal subfield atrophy in mild cognitive impairment. Transl Stroke Res. 2021;12(2):284-92. https://doi. org/10.1007/s12975-020-00847-4.

43. Grau-Olivares M, Arboix A, Bartrés-Faz D, Junqué C. Neuropsychological abnormalities associated with lacunar infarction. J Neurol Sci. 2007;257(12):160-5. https://doi.org/10.1016/j.jns.2007.01.022.

\section{Publisher's Note}

Springer Nature remains neutral with regard to jurisdictional claims in published maps and institutional affiliations.
Ready to submit your research? Choose BMC and benefit from:

- fast, convenient online submission

- thorough peer review by experienced researchers in your field

- rapid publication on acceptance

- support for research data, including large and complex data types

- gold Open Access which fosters wider collaboration and increased citations

- maximum visibility for your research: over 100M website views per year

At BMC, research is always in progress.

Learn more biomedcentral.com/submissions 